\title{
Network and biosignature analysis for the integration of transcriptomic and metabolomic data to characterize leaf senescence process in sunflower
}

\author{
Sebastián Moschen ${ }^{1,2}$, Janet Higgins ${ }^{3}$, Julio A. Di Rienzo ${ }^{4}$, Ruth A. Heinz ${ }^{1,2}$, Norma Paniego ${ }^{1,2}$
} and Paula Fernandez $1,2,5^{*}$

From Statistical Methods for Omics Data Integration and Analysis 2014

Heraklion, Crete, Greece. 10-12 November 2014

\begin{abstract}
Background: In recent years, high throughput technologies have led to an increase of datasets from omics disciplines allowing the understanding of the complex regulatory networks associated with biological processes. Leaf senescence is a complex mechanism controlled by multiple genetic and environmental variables, which has a strong impact on crop yield. Transcription factors (TFs) are key proteins in the regulation of gene expression, regulating different signaling pathways; their function is crucial for triggering and/or regulating different aspects of the leaf senescence process. The study of TF interactions and their integration with metabolic profiles under different developmental conditions, especially for a non-model organism such as sunflower, will open new insights into the details of gene regulation of leaf senescence.
\end{abstract}

Results: Weighted Gene Correlation Network Analysis (WGCNA) and BioSignature Discoverer (BioSD, Gnosis Data Analysis, Heraklion, Greece) were used to integrate transcriptomic and metabolomic data. WGCNA allowed the detection of 10 metabolites and 13 TFs whereas BioSD allowed the detection of 1 metabolite and 6 TFs as potential biomarkers. The comparative analysis demonstrated that three transcription factors were detected through both methodologies, highlighting them as potentially robust biomarkers associated with leaf senescence in sunflower.

Conclusions: The complementary use of network and BioSignature Discoverer analysis of transcriptomic and metabolomic data provided a useful tool for identifying candidate genes and metabolites which may have a role during the triggering and development of the leaf senescence process. The WGCNA tool allowed us to design and test a hypothetical network in order to infer relationships across selected transcription factor and metabolite candidate biomarkers involved in leaf senescence, whereas BioSignature Discoverer selected transcripts and metabolites which discriminate between different ages of sunflower plants. The methodology presented here would help to elucidate and predict novel networks and potential biomarkers of leaf senescence in sunflower.

Keywords: WGCNA, BioSignature Discoverer, Leaf senescence, Transcriptomic, Metabolomic, Data integration, Sunflower

\footnotetext{
* Correspondence: fernandez.pc@inta.gob.ar

${ }^{1}$ Instituto de Biotecnología, Centro de Investigaciones en Ciencias

Agronómicas y Veterinarias, Instituto Nacional de Tecnología Agropecuaria,

Hurlingham, Buenos Aires, Argentina

${ }^{2}$ Consejo Nacional de Investigaciones Científicas y Técnicas, Ciudad

Autónoma de Buenos Aires, Argentina

Full list of author information is available at the end of the article
} 


\section{Background}

Functional genomics is a field of molecular biology that studies the functions and interactions between genes and proteins from large datasets generated by genome projects. Over recent years, the development of new high throughput technologies among different omics approaches has led to the availability of a large volume of transcriptomic, metabolomic, proteomic, physiological and phenotypic data. In this sense, the multidisciplinary synergy between molecular biology, statistics and informatics emerge as necessary to support the use and interpretation of the results in the area of functional genomics in order to provide specific tools for the simultaneous analysis of many genes.

Senescence is the final stage of leaf development and precedes cell death. Once the senescence program is triggered, important highly regulated gene expression changes occur, leading to important changes in the metabolism [1-7].

In sunflower, which is the fourth most important oil crop worldwide, a delay in leaf senescence has a strong impact on yield; this effect has also been shown in other crops. Maintaining the photosynthetic leaf area especially during the reproductive stage [8-12] has been shown to impact on the gap between potential and real yield observed, this is due to the incapacity of plants to maintain their green leaf area for longer periods [13, 14].

Transcription factors (TFs) are key proteins involved in the regulation of gene expression and signal transduction networks, regulating different biological processes. Several transcription factor families have been associated with leaf senescence in a range of species [15-29] and their function is crucial for triggering and/or regulating the different aspect of this process.

Various software tools have been developed with the aim of analyzing datasets to predict hub genes which could regulate a specific pathway or be involved in a particular biological process. Weighted Gene Correlation Network Analysis (WGCNA) is an R package method designed to find clusters (modules) of highly correlated genes or metabolites. WGCNA calculates a Pearson's correlation matrix for all the genes and then transforms the correlation matrix into an adjacency matrix by raising all values to a soft threshold power; this has the effect of emphasizing the strong correlations and penalizing the weaker correlations. The module eigengene $(M E)$ is the first principal component of a given module and can be considered as representative of the module's gene expression profile. Modules often represent specific biological processes; highly connected hub genes within the module are often regulatory genes and represent candidate biomarker. WGCNA can be used to construct networks in which each node represents a gene or metabolite and the connecting lines (edges) represent correlations between the nodes. WGCNA has been widely used as a method to cluster gene expression and metabolite data and identify hub biomarker genes in several non-model plant species such as tomato [30], Brassica [31], Petunia [32], rice [33] and Ficus [34].

BioSignature Discoverer (BioSD) is a software application devised for identifying molecular signatures in different biological datasets, such as Next Generation Sequencing, microarray data and metabolic profiles, in a statistically robust, computationally efficient, and userfriendly way. A signature is defined as the minimal set of molecular quantities that collectively yield maximal predictive performance. Thus, a signature does not contain irrelevant or redundant quantities, given the selected ones. In addition, there may be numerous equivalent signatures that lead to equally accurate predictions, employing different molecular quantities. The core of this application uses a feature selection algorithm that belongs to the class of Bayesian Network, constraintbased learning [35] and that is able to identify multiple, statistically equivalent signatures.

In this work we used these two complementary methods, WGCNA and BioSD, with the aim of identifying potential transcription factors and metabolites, as putative biomarkers associated with leaf senescence in sunflower.

\section{Methods}

\section{Plant material and experimental conditions}

The sunflower leaf senescence assays were conducted under field conditions. The experiment was carried out at the INTA Balcarce Experimental Station $\left(37^{\circ} 45^{\prime} \mathrm{S}\right.$, $\left.58^{\circ} 18^{\prime} \mathrm{W}\right)$ as previously described $[36,37]$. The experiment was sowed during the 2010/11 growing season. The sunflower hybrid VDH 487 (Advanta Seeds, Argentina) was sown at a 7.2 plants $/ \mathrm{m} 2$ with three biological replicates (plots), each one consisted of three randomly selected plants from each plot.

Sunflower assay was conducted under control conditions, without limitations (water and nutrients). Transcriptomic and metabolomics profiling was performed using the leaf 10 (numbered from the bottom to the top of the plant) at three different development stages labeled as T-0 (young leaf, 48 days after emergence of the plant with maximum chlorophyll content), T-1 (pre-anthesis leaf, 62 days after emergence of the plant with $80 \%$ of chlorophyll content) and T-2 (post-anthesis leaf, with senescence symptoms, 69 days after emergence of the plant with $50 \%$ of chlorophyll content) $[36,37]$. The samples were immediately frozen in liquid nitrogen upon collection and saved at $-80{ }^{\circ} \mathrm{C}$ until processing. High quality total RNA was isolated from $100 \mathrm{mg}$ of frozen tissue using TriPure, according to the manufacturer's instructions (Roche, Buenos Aires, Argentina). The genomic DNA was 
eliminated by treatment with DNase I for $20 \mathrm{~min}$ at room temperature (Invitrogen, Buenos Aires, Argentina). The RNA concentration was measured using a Nanodrop ND-1000 spectrophotometer (NanoDrop Technologies, Wilmington, Delaware USA). The purity and integrity of total RNA was determined by $260 / 280 \mathrm{~nm}$ ratio and by NanoBioanalyzer RNA-6000 analysis (Agilent Technologies, Palo Alto, California USA).

\section{Data set}

Transcriptomic profiling was performed using a custom sunflower microarray (Agilent $4 \times 44 \mathrm{~K}$ format) which has been previously described [38]. Background correction was performed using the rma algorithm from the backgroundCorrect function (offset $=16$, other parameters by default). Between arrays normalization was performed using the quantile method from the normalizeBetweenArray function. Finally, gene expressions were transformed to $\log _{2}$ scale and information from technical replicates was incorporated by calculating the median parameter. Statistically significant probesets where identified using the lmFit and contrasts.fit functions from the R software Limma Bioconductor Package [39, 40] A total of 4,909 probes were shown to be statistically significant during leaf senescence as the plants develop (T-1 vs. T-0 and/or T-2 vs.T-0). The statistical parameters were $\mathrm{p}$-value lower than 0.05 and fold-change higher or lower than 2 [37]. Sunflower transcription factors (TFs) were identified by comparing approximately 23,000 TFs sequences from Arabidopsis lyrata, Arabidopsis thaliana, Oryza sativa, Populus trichocarpa, Vitis vinifera and Zea mays available from the Plant Transcription Factor Database (http://plntfdb.bio.uni-potsdam.de/v3.0/) [41] with SUR v1.0 database [38] using Blast software. A total of 82 TFs were differentially expressed during leaf senescence.

Metabolic profiling was performed using the GC-TOFMS system (LECO Corporation, St. Joseph, Michigan, USA). Metabolite extraction was performed by promoting the extraction of lipophilic and polar compounds according to recently published protocols [42] adapted for sunflower tissue samples [43]. The chromatograms and spectra were evaluated using ChromaTOF (LECO Corporation, St. Joseph, Míchigan, USA) and TagFinder [44]. Ion spectra were compared to the Golm Metabolome Database (http://gmd.mpimp-golm.mpg.de/). Metabolite levels were normalized to fresh weight using ribitol as the internal control. Changes in metabolite levels along leaf development were calculated as foldchange relative to the first sampled time (T-0).

\section{Weighted Gene Co-expression network analysis (WGCNA)} WGCNA was performed using the WGCNA R package (v1.42) as described by Langfelder and Horvath [45]. The expression values for 9,592 non-redundant genes and levels of 62 metabolites for the 9 samples were used to construct two separate networks. Samples were clustered using the function hclust to check that there were no outliers. The pickSoftThreshold function was used to select the soft threshold power used to construct a network based on the criterion of approximate scale-free topology. The power value selected was the lowest power for which the scale-free topology fit index curve flattened out upon reaching a high value. The next step was to transform the adjacency matrix into a topological overlap matrix (TOM), which summarizes the degree of shared connections between any two genes. The TOM matrix was then converted into a dissimilarity matrix. Genes were then clustered using the average linkage hierarchical clustering and the modules were identified in the resulting dendrogram using the dynamic hybrid tree cut method (Additional file 1). Found modules were trimmed of genes whose correlation with the module eigengene (KME) was less than minKMEtoStay. If p-values of the higher correlations were smaller than those of the native module by the factor reassignThreshold, the gene was re-assigned to the closer module. Modules whose eigengenes were highly correlated were merged. This was achieved by clustering module eigengenes using the dissimilarity given by one minus their correlation, cutting the dendrogram at the height mergeCutHeight and merging all modules on each branch below mergeCutHeight. This was implemented using the blockwiseModules function in WGCNA which performs the network construction and consensus module detection. The following settings were used for the gene network; power $=6$, minModuleSize $=50$, mergeCutHeight $=0.2$, maxBlockSize $=10000$, deepSplit $=2$, reassignThreshold $=$ $1 \mathrm{e}-6$ and $\operatorname{minKMEtoStay}=0.5$, networkType $=$ "unsigned", TOMType $=$ "signed". The genes were divided into 17 modules; the module sizes ranged from 99 to 2,967 genes, 501 genes were not assigned to any of the modules. The following settings were used for the metabolite network; power $=10, \quad$ minModuleSize $=5, \quad$ mergeCutHeight $=0.2$, maxBlockSize $=1000$, deepSplit $=2$, reassignThreshold $=$ $1 \mathrm{e}-6$ and $\operatorname{minKMEtoStay}=0.5$, networkType $=$ "unsigned", TOMType = "signed". The metabolites were divided into 3 modules; the module sizes ranged from 10 to 35 metabolites, 5 metabolites were not assigned to any of the modules.

Module Membership (MM) is a quantitative measure of module membership; this is the correlation of the ME and the gene expression profile for each gene in the module. The gene with the highest connectivity in each module was returned using the function chooseTopHubInEachModule.

The networks were exported from WGCNA using the function exportNetworkToCytoscape.

The gene network showed approximate scale free topology, whereas the metabolic network did not satisfy scale 
free topology: 9592 genes scaleFreeRsquared 0.86 slope -0.62 and 62 metabolites scaleFreeRsquared 0.33 slope -0.54 (Additional file 2).

A total of 24 metabolites with a degree higher than 35 were then selected as metabolite hubs and correlated with the list of 82 TFs statistically significant during senescence (Additional file 3) using Pearson correlation. Correlations with p-value $<0.0001$ were selected and visualized using Cytoscape [46].

\section{BioSignature Discoverer}

The statistical analysis pipeline of BioSD, a plugin of QIAGEN's CLC-Bio workbench (CLC Bio, Aarhus, Denmark), has been used for identifying gene-expression signatures able to discriminate the age of sunflower plants. Particularly, the plugin has been applied on the expression values of 82 transcription factors that were found differentially expressed during leaf senescence, on the 62 metabolites measured in the study, and on the combination of these two data types. Additionally, the pipeline has been separately applied on the extended list of 9,592 non-redundant genes.

BioSD employs a complex machine-learning approach in order to discover such signatures and for quantifying their actual predictive power. Particularly, this tool employs novel, proprietary feature selection algorithms to identify the signatures, inspired by prior work on Bayesian-Network constraint-based learning [35]. Notably, the pipeline is also able to identify multiple equivalent signatures, whose predictive performances are statistically equivalent. To avoid under fitting, the tool automatically tries multiple algorithms for feature selection and prediction modeling, as well as multiple values for tuning them. The tool also employs sophisticated methods, based on an enhanced version of cross-validation [47], for providing conservative estimations of the performances that can be achieved with the given signatures.

The BioSD interface allows the user to modulate the trade-off between quality of the results and computational requirements through the 'Tuning Effort' parameter. This parameter regulates the extent to which the tool will attempt tuning the feature selection and prediction modeling algorithms. In all analyses, the 'Tuning Effort' was set to 'Extensive', in order to favor result accuracy at the expense of higher computational time.

\section{Results and discussion}

WGCNA is a powerful method used to identify clusters of highly correlated genes which are potentially coregulated. All the connections for the metabolites with an edge weight $>0.1$ produced a network of 51 nodes and 765 edges. The connections between the 82 significant differentially expressed transcription factors were selected from the gene network with an edge weight $>0.4$, producing a network of 75 nodes and 1202 edges.

The networks were visualized in Cytoscape by edge weight and degree (Fig. 1a and b).

A total of 20 transcription factors and 24 metabolites showed the highest number of connections (degree), over $80 \%$ of the maximum number of connections observed (degree $>60$ for TFs and degree $>35$ for metabolites), highlighting them as potentially co-regulated hub biomarkers during the senescence process in sunflower.

Pearson correlation analysis between the 24 hub metabolites and the list of 82 TFs significant during senescence was performed in order to integrate both analyses. The resulting network contained 74 nodes and 455 edges (Fig. 2).

The integration results showed that 7 metabolite hubs correspond to sugars and TCA cycle metabolites(from a total of 10) and the NAC TFs family, widely reported as senescence associated, showed 3 members (from a total of 13) as hub genes.

On the other hand, BioSD allows identification of the most informative genes and metabolites for discriminating between the various stages of the leaf senescence process. Table 1 lists all the transcription factors and metabolites whose values allow the predicting of senescence in sunflower plants. All these quantities have equivalent discriminative power and $100 \%$ stability, which mean that they are consistently selected even when other datasets are included.

Table 2 reports the 'in-sample' and 'out-of-sample' performances of the selected transcripts and metabolites. The in-sample values quantify the fitness of the predictive models, and poor results would indicate presence of outliers, unmeasured yet needed predictive factors, or poorly modeled trends in the data. The out-of-sample values estimate the performances that are expected if the selected expression and metabolic values are used for predicting the age of new, independent samples of sunflower plants (provided the new samples come from the same population of the training data). Assuming $y$ and $\hat{y}$ are the observed and predicted age of $n$ plants. The $R^{2}$ metric measures the proportion of $y$ variance explained by the predictive model, $R^{2}$ values close to 1 are indicative of good fit, while the Mean Absolute Error (MAE) and Mean Squared Error (MSE) statistics quantify predictions' deviations from the actual age as $M A E=\frac{1}{n} \cdot \sum a b s(y-\hat{y})$ and $M S E=\frac{1}{n} \cdot \sum(y-\hat{y})^{2}$.

In the present application both in- and out-of-sample performances show quite good results, indicating (a) that the predictive models adequately fit the available samples and (b) that the selected transcript factors and metabolites have a high predictive power. MAE, a robust estimator of the standard deviation, is around 2.5 days for the "out of 


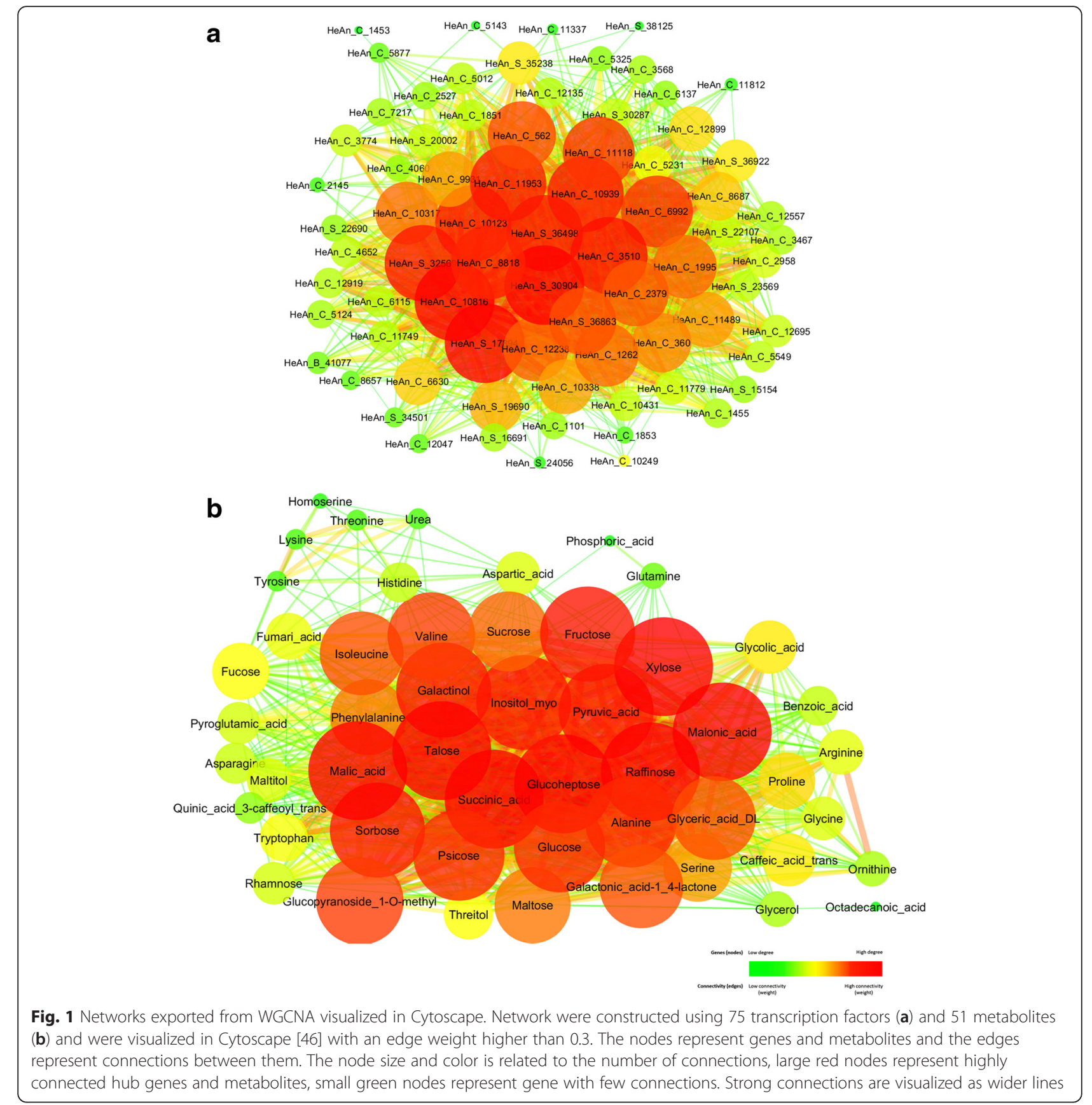

sample" for both, metabolite and transcript data, meaning that it should be possible to predict the age of a new plant on the basis of transcriptional or metabolic information with an average error of approximately three days.

Residual vs. predicted value plots did not showed any apparent pattern or trend, indicating that the models have fitted the data adequately.

The analysis of the complete list of gene (9,592 nonredundant sunflower genes) integrated with metabolic information identified twelve distinct signatures each composed of two elements. Particularly, each signature contains the gene HeAn_C_267, coupled either with the Alanine metabolite or with one of the following eleven genes: HeAn_C_1048, HeAn_C_11045, HeAn C_11058, HeAn_C_11653, HeAn_C_243, HeAn_C 3359, HeAn_C_8838, HeAn_S_19086, HeAn_S_20155, HeAn_S_30642, HeAn_S_35632. These signatures have slightly better performances than the single biomarkers reported in Table 1 (see Additional file 4).

These results indicate that the Alanine metabolite is interchangeable, in terms of predictive power with each of the other eleven genes. Thus, practitioners interested 


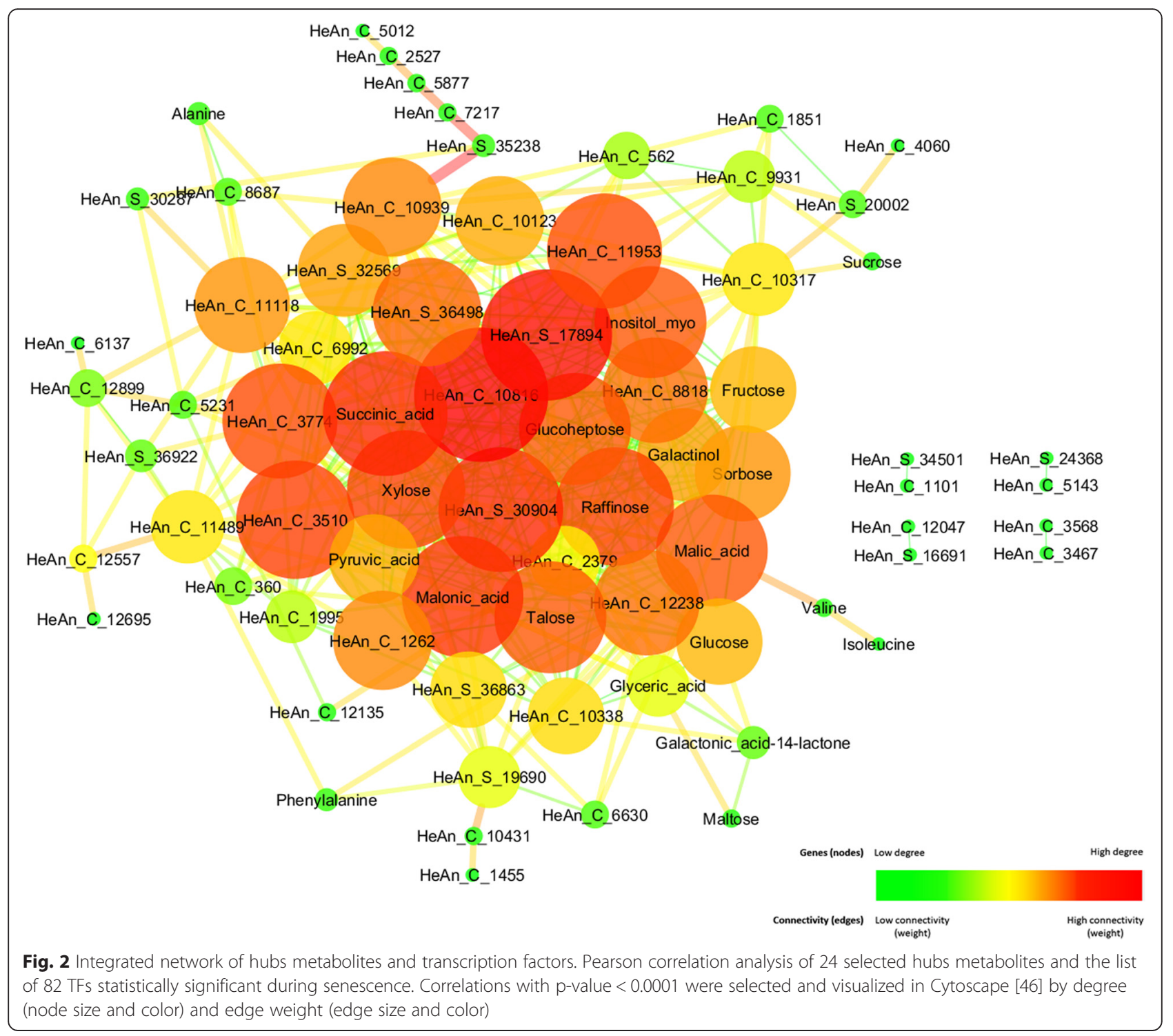

Table 1 List of biosignatures detected in each dataset

\begin{tabular}{lll}
\hline Metabolites & Transcription factors & Integrated list \\
\hline Alanine & HeAn_C_10939 & Alanine \\
& HeAn_C_11118 & HeAn_S_15792 \\
& HeAn_C_11953 & HeAn_C_10939 \\
& HeAn_C_12899 & HeAn_S_38523 \\
& HeAn_C_8526 & HeAn_C_9238 \\
& HeAn_C_9238 & HeAn_S_32569 \\
& HeAn_S_15792 & HeAn_C_11118 \\
& HeAn_S_32569 & \\
& HeAn_S_36498 & \\
HeAn_S_38523 &
\end{tabular}

Each signature is composed of a single biomarker, and all signatures are expected to have the same predictive performance (see Table 2) in deploying this signature in practice (e.g., researchers, agronomists) can freely decide whether to build a predictive model using exclusively transcriptional information or a mixture of transcriptional and metabolic data. This decision can depend on multiple factors, particularly considerations about the cost and technical practicability of measuring the quantities included in the signature.

In recent years, many studies have focused on the detection of different genes up or down-regulated along the progression of the leaf senescence process, identifying TFs that could be triggering the process $[1,3,29$, 48-51]. Hence, studying interactions between these TFs under different conditions, for a non-model organism, will open new insights into the details of gene regulation by identifying new interactions and comparing them to those already observed in model organisms [52]. 
Table 2 Predicted performances of the selected biosignatures

\begin{tabular}{|c|c|c|c|c|}
\hline & Metric & In Sample & Out Sample & $95 \%$ Confidence Interval \\
\hline \multirow[t]{3}{*}{ Metabolites } & $R^{2}$ & 0.997 & 0.840 & {$[0.335,0.972]$} \\
\hline & Mean Absolute Error & 0.228 & 2.503 & {$[0.783,4.060]$} \\
\hline & Mean Squared Error & 0.2104 & 12.180 & {$[0.964,24.916]$} \\
\hline \multirow[t]{3}{*}{ Transcription factors } & $R^{2}$ & 0.983 & 0.825 & {$[0.565,0.939]$} \\
\hline & Mean Absolute Error & 0.897 & 2.658 & {$[1.009,4.950]$} \\
\hline & Mean Squared Error & 1.239 & 13.315 & {$[3.743,29.804]$} \\
\hline \multirow[t]{3}{*}{ Integrated list } & $R^{2}$ & 0.9965 & 0.909 & {$[0.721,0.986]$} \\
\hline & Mean Absolute Error & 0.465 & 1.779 & {$[0.487,3.919]$} \\
\hline & Mean Squared Error & 0.2639 & 6.927 & {$[0.469,18.832]$} \\
\hline
\end{tabular}

Performances are reported in terms of the determination coefficient $\mathrm{R}^{2}$, Mean Absolute Error (MAE) and Mean Squared Error (MSE, see text for more details on these metrics). The in-sample performances quantify the fitness of the predictive models on the training data, while the out-of-sample values estimate the expected performance on new data. Confidence intervals are calculated using a bootstrap approach

One of the most important aims in new generation biotech crops is to increase the yield and to improve tolerance to different stresses. Although sequencing-based approaches are becoming the method of choice for gene expression profiling compared to hybridization-based ones, for non-model species for which a reference sequence is not available, the hybridization approach can still be the more reliable method [53].

This study reports on the use of two complementary software applications with the aim of producing a powerful network and predicting potential biosignatures enabling the identification of important TFs and metabolites involved during the leaf senescence process in sunflower.

Using WGCNA analysis, genes and metabolites are clustered in one or more modules. Genes in each module are highly correlated, and consequently they are likely to be co-regulated during the senescence process. BioSD identifies which genes are the most informative in order to discriminate between different stages of leaf development. The selected genes, in this case, are not necessarily correlated among themselves.

In other words, WGCNA attempts to identify the elements involved in the senescence process, and to gain insight of their interplay. On the other hand, BioSD tries to retain the minimum number of these elements that are needed for optimal prediction of the plant senescence process.

WGCNA allowed the detection of metabolite hubs during leaf senescence, most of them correspond to sugars and TCA cycle metabolites. Sugars are central elements in the sink-source relationships $[54,55]$ and have been reported as growth [56] and photosynthesis regulators [57]. Sunflower is a crop plant with a strong demand for nutrients, especially sugar as a substrate in the oil synthesis during grain filling; this could potentially act as a trigger signal for the senescence process.
BioSD allowed the detection of one metabolite and ten TFs as potential biomarkers. The selected biosignatures are able to estimate the senescence of a plant with an error of approximately 2.5 days. When both TF and metabolite data are simultaneously analyzed, the expected error decreases to 1.8 days, indicating that the integration of different omic data allows better predictions of sunflower senescence by BioSD analysis.

Transcription factors are major players and some of them constitute major hubs in signaling pathways. In both analyses, the selected genes belong to transcription factor families widely reported as senescence associated in model species.

When we analyze the integration of metabolites and TFs (Table 3) we found that three TFs (HeAn_C_10939, HeAn_C_11118 and HeAn_S_32569) are detected as biomarkers independently in the two methods. The expression profiles of the selected biomarkers during leaf senescence are displayed in the Additional file 5 . HeAn_C_10939 presents high sequence similarity to ATERF3 (AT1G50640) which encodes a member of the ERF (ethylene response factor).

Transgenic Arabidopsis plants with enhanced expression of this TF showed precocious leaf senescence [25]. Additionally, $H e A n_{-} C_{-} 11118$ has high sequence similarity to AtNAP/ANAC029 (AT1G69490), a NAC transcription factor which acts downstream of EIN2 and EIN3 genes. EIN3 positively regulates leaf senescence by activating ORE1 and AtNAP. Genetic analysis suggest that both genes act in distinct and overlapping signaling pathways regulating leaf senescence in Arabidopsis [17, 58]. HaNAC01, a putative sunflower orthologous gene to ORE1, has previously been reported in sunflower as a candidate biomarker for leaf senescence [36], which reinforces these genes as potential regulators of the senescence process in sunflower. 
Table 3 Candidate biomarkers detected by the two complementary methods, WGCNA and BioSignature Discoverer

\begin{tabular}{|c|c|c|c|c|c|}
\hline \multicolumn{3}{|l|}{ WGCNA } & \multicolumn{3}{|l|}{ Biosignatures } \\
\hline Sunflower ID & Arabidopsis ID & TF Family & Sunflower ID & Arabidopsis ID & TF Family \\
\hline HeAn_C_10816 & AT1G52890 & NAC & HeAn_S_15792 & AT5G26210 & Alfin-like \\
\hline HeAn_S_17894 & AT5G55580 & mTERF & HeAn_C_10939 & AT1G50640 & AP2 \\
\hline HeAn_C_3510 & AT4G27410 & NAC & HeAn_S_38523 & AT3G16770 & AP2 \\
\hline HeAn_S_30904 & AT4G17810 & $\mathrm{C} 2 \mathrm{H} 2$ & HeAn_C_9238 & AT2G39770 & GRAS \\
\hline HeAn_C_3774 & AT4G02590 & bHLH & HeAn_S_32569 & AT5G49620 & MYB \\
\hline HeAn_C_11953 & AT2G45650 & MADS & HeAn_C_11118 & AT1G69490 & NAC \\
\hline HeAn_S_36498 & AT2G30400 & OFP & Alanine & & \\
\hline HeAn_C_10939 & AT1G50640 & AP2 & & & \\
\hline HeAn_C_1262 & AT1G64860 & Sigma70-like & & & \\
\hline HeAn_C_8818 & AT2G28810 & C2C2-Dof & & & \\
\hline HeAn_C_11118 & AT1G69490 & NAC & & & \\
\hline HeAn_C_12238 & AT1G27320 & Orphans & & & \\
\hline HeAn_S_32569 & AT5G49620 & MYB & & & \\
\hline \multicolumn{6}{|l|}{ Succinic_acid } \\
\hline \multicolumn{6}{|l|}{ Malonic_acid } \\
\hline \multicolumn{6}{|l|}{ Raffinose } \\
\hline \multicolumn{6}{|l|}{ Xylose } \\
\hline \multicolumn{6}{|l|}{ Talose } \\
\hline \multicolumn{6}{|l|}{ Inositol_myo } \\
\hline \multicolumn{6}{|l|}{ Glucoheptose } \\
\hline \multicolumn{6}{|l|}{ Malic_acid } \\
\hline \multicolumn{6}{|l|}{ Galactinol } \\
\hline Sorbose & & & & & \\
\hline
\end{tabular}

Biomarkers from WGCNA were selected based on their degree (higher than 20) and biomarkers from BioSignature Discoverer correspond to the analysis of the integrated list. Biomarkers detected independently in the two methods are in bold

HeAn_S_32569 has high sequence similarity to MYB78 (AT5G49620). This TF has not yet been directly associated with the senescence process, however, members of this family have been reported as associated to senescence and involved in response to several abiotic stresses [21, 22, 59].

These results suggest that WGCNA and the identification of molecular biosignatures are powerful tools for the detection of potential biomarkers. However, it should be noted that these results are obtained on a relatively small experiment (3 biological replicates for 3 treatments). Further studies would be advisable in order to better estimate the predictive performance of this approach and to achieve best fit for the list of candidate omics signatures.

The implementation of data integration analysis associated to different biotic and abiotic stresses is a powerful tool, especially in a non-model crop such as sunflower for which the complete genome sequence is not yet available. These results open new strategies of analysis to explore and detect potential biomarkers associated with leaf senescence that will be useful for future molecular breeding programs.

\section{Conclusions}

The complementary analysis of transcriptomic and metabolomic data with WGCNA and BioSD emerge as a useful strategy to predict not only the age of the plants but also to detect and identify potential biomarkers associated with leaf senescence. In the case of sunflower, a worldwide oil crop, the implementation of WGCNA enabled the construction of a hypothetical network which was used to infer relationships between TFs and metabolites and the identification of hubs as potential candidate biomarkers involved in leaf senescence, whereas BioSD selected transcripts and metabolites to discriminate the age of sunflower plants. This methodological approach is a novel strategy for omics data integration, it can be used to elucidate and predict novel networks as well as identifying potential transcripts as putative biomarker hub genes at different developmental stages. 


\section{Additional files}

Additional file 1: Cluster dendrogram visualizing the modules from WGCNA. a Genes, b Metabolites were clustered using the average linkage hierarchical clustering and modules identified in the resulting dendrogram by the dynamic hybrid tree cut method. (DOCX $530 \mathrm{~kb}$ )

Additional file 2: Scale-free topologies. a Genes, b Metabolites. The left panel shows a histogram of network connectivity. The right panel shows a log-log plot of the histogram. The approximate straight line relationship (high R2 value) shows approximate scale free topology. (DOCX 143 kb)

Additional file 3: List of 24 hubs metabolites and 82 transcription factors. The list shows the identifiers and the normalized expression data of the different samples. (XLSX $21 \mathrm{~kb}$ )

Additional file 4: BioSignature Discoverer results on predicting senescence from integrated metabolomics and transcriptomics data in sunflower. (DOCX $38 \mathrm{~kb})$

Additional file 5: Expression profiles of the selected biomarkers during leaf senescence. The list shows the identifiers, the expression profile of candidate biomarkers in log2 scale and the standardization of this expression profile. The figures show the genes and the metabolites in red and blue lines respectively. (XLSX 27 kb)

\section{Competing interests}

The authors declare that they have no competing interests.

\section{Authors' contributions}

PF and SM conceived and designed the work, contributed to the implementation of WGCNA for the data, tested software to visualize and understand the data analysis and wrote the first draft of the manuscript, $\mathrm{JH}$ implemented WGCNA and tested different parameters to optimize the network design, JADR revised the manuscript and statistically supported software analysis, $\mathrm{RH}$ and NP initiated the project and contributed to the work by the interpretation, discussion of the data and critically revised the manuscript. All authors read and approved the final manuscript.

\section{Acknowledgements}

We want to thank Gnosis Data Analysis (http://www.gnosisda.gr/) for their support in applying BioSignature Discoverer.

\section{Declarations}

Publication charges for this work were funded by INTA Préstamo BID PICT 20120390 and PIP CONICET 11220120100262 CO.

This article has been published as part of BMC Bioinformatics Volume 17 Supplement 5, 2016: Selected articles from Statistical Methods for Omics Data Integration and Analysis 2014. The full contents of the supplement are available online at http://bmcbioinformatics.biomedcentral.com/articles/ supplements/volume-17-supplement-5.

\section{Author details}

${ }^{1}$ Instituto de Biotecnología, Centro de Investigaciones en Ciencias Agronómicas y Veterinarias, Instituto Nacional de Tecnología Agropecuaria, Hurlingham, Buenos Aires, Argentina. ${ }^{2}$ Consejo Nacional de Investigaciones Científicas y Técnicas, Ciudad Autónoma de Buenos Aires, Argentina. ${ }^{3}$ The Genome Analysis Centre, Norwich Research Park, Norwich NR4 7UH, UK ${ }^{4}$ Facultad de Ciencias Agropecuarias, Universidad Nacional de Córdoba, Córdoba, Argentina. ${ }^{5}$ Escuela de Ciencia y Tecnología, Universidad Naciona de San Martín, San Martín, Buenos Aires, Argentina.

\section{Published: 6 June 2016}

\section{References}

1. Buchanan-Wollaston V, Earl S, Harrison E, Mathas E, Navabpour S, Page T, Pink D. The molecular analysis of leaf senescence-a genomics approach. Plant Biotechnol J. 2003:1:3-22.

2. Gan S. Mitotic and postmitotic senescence in plants. Sci Aging Knowl Env. 2003:2003(38):RE7.

3. Gepstein S, Sabehi G, Carp M-JM-J, Hajouj T, Nesher MFO, Yariv I, Dor C, Bassani M. Large-scale identification of leaf senescence-associated genes. Plant J. 2003;36:629-42.
4. Guo Y, Gan S. Leaf senescence: signals, execution, and regulation. Curr Top Dev Biol. 2005;71:83-112

5. Lim PO, Kim HJ, Nam HG. Leaf Senescence. Annu Rev Plant Biol. 2007;58:115-36.

6. Weaver LM, Gan S, Quirino B, Amasino RM. A comparison of the expression patterns of several senescence-associated genes in response to stress and hormone treatment. Plant Mol Biol. 1998:37:455-69.

7. Quirino BF, Noh YS, Himelblau E, Amasino RM. Molecular aspects of leaf senescence. Trends Plant Sci. 2000;5:278-82.

8. Sadras VO, Quiroz F, Echarte L, Escande A, Pereyra VR. Effect of Verticillium dahliae on photosynthesis, leaf expansion and senescence of field-grown sunflower. Ann Bot. 2000;86:1007-15.

9. Sadras VO, Echarte L, Andrade FH. Profiles of leaf senescence during reproductive growth of sunflower and maize. Ann Bot. 2000;85:187-95.

10. Kusaba M, Tanaka A, Tanaka R. Stay-green plants: what do they tell us about the molecular mechanism of leaf senescence. Photosynth Res. 2013;117:221-34.

11. De la Vega AJ, Cantore MA, Sposaro MM, Trápani N, López Pereira M, Hall AJ. Canopy stay-green and yield in non-stressed sunflower. F Crop Res. 2011;121:175-85.

12. Gregersen PL, Culetic A, Boschian L, Krupinska K. Plant senescence and crop productivity. Plant Mol Biol. 2013;82:603-22.

13. Dosio GAA, Aguirreza LAN, Andrade FH, Pereyra VR, Aguirrezábal LAN Solar radiation intercepted during seed filling and oil production in two sunflower hybrids. Crop Sci. 2000;1644:1637-44.

14. Aguirrezábal LAN, Lavaud Y, Dosio GAA, Izquierdo NG, Andrade FH, González LM. Weight per seed and oil concentration in a sunflower hybrid are accounted for by intercepted solar radiation during a definite period of seed filling. Crop Sci. 2003;43:152-61.

15. Balazadeh S, Wu A, Mueller-Roeber B. Salt-triggered expression of the ANAC092-dependent senescence regulon in Arabidopsis thaliana. Plant Signal Behav. 2010;5:733.

16. Balazadeh S, Kwasniewski M, Caldana C, Mehrnia M, Zanor MI, Xue G-PP, Mueller-Roeber B. ORS1, an H2O2-responsive NAC transcription factor, controls senescence in Arabidopsis thaliana. Mol Plant. 2011:4:346-60.

17. Guo Y, Gan S. AtNAP, a NAC family transcription factor, has an important role in leaf senescence. Plant J. 2006:46:601-12.

18. Kim JH, Woo HR, Kim J, Lim PO, Lee IC, Choi SH, Hwang D, Nam HG. Trifurcate feed-forward regulation of age-dependent cell death involving miR164 in Arabidopsis. Science. 2009;323:1053-7.

19. Kim Y-S, Sakuraba Y, Han S-H, Yoo S-C, Paek N-C. Mutation of the Arabidopsis NAC016 transcription factor delays leaf senescence Plant Cell Physiol. 2013;54:1660-72

20. Wang Z, Dane F. NAC ( NAM / ATAF / CUC ) transcription factors in different stresses and their signaling pathway. Acta Physiol Plant. 2013:35:1397-408.

21. Zhang X, Ju H-W, Chung M-S, Huang P, Ahn S-J, Kim CS. The R-R-type MYBlike transcription factor, AtMYBL, is involved in promoting leaf senescence and modulates an abiotic stress response in Arabidopsis. Plant Cell Physiol. 2011;52:138-48

22. Jaradat MR, Feurtado JA, Huang D, Lu Y, Cutler AJ. Multiple roles of the transcription factor AtMYBR1/AtMYB44 in ABA signaling, stress responses, and leaf senescence. BMC Plant Biol. 2013;13:192.

23. Chen G-H, Liu C-P, Chen S-CG, Wang L-C. Role of ARABIDOPSIS A-FIFTEEN in regulating leaf senescence involves response to reactive oxygen species and is dependent on ETHYLENE INSENSITIVE2. J Exp Bot. 2012;63:275-92.

24. Chen G-H, Chan Y-L, Liu C-P, Wang L-C. Ethylene response pathway is essential for ARABIDOPSIS A-FIFTEEN function in floral induction and leaf senescence. Plant Signal Behav. 2012;7:457-60.

25. Koyama T, Nii H, Mitsuda N, Ohta M, Kitajima S, Ohme-Takagi M, Sato F. A regulatory cascade involving class II ETHYLENE RESPONSE FACTOR transcriptional repressors operates in the progression of leaf senescence. Plant Physiol. 2013;162:991-1005.

26. Ulker B, Shahid Mukhtar M, Somssich IE. The WRKY70 transcription factor of Arabidopsis influences both the plant senescence and defense signaling pathways. Planta. 2007:226:125-37.

27. Besseau S, Li J, Palva ET. WRKY54 and WRKY70 co-operate as negative regulators of leaf senescence in Arabidopsis thaliana. J Exp Bot. 2012;63:2667-79.

28. Rushton DL, Tripathi P, Rabara RC, Lin J, Ringler P, Boken AK, Langum TJ, Smidt L, Boomsma DD, Emme NJ, Chen X, Finer JJ, Shen QJ, Rushton PJ. WRKY transcription factors: key components in abscisic acid signalling. Plant Biotechnol J. 2012;10:2-11. 
29. Balazadeh S, Riaño-Pachón DM, Mueller-Roeber B. Transcription factors regulating leaf senescence in Arabidopsis thaliana. Plant Biol. 2008;10 Suppl 1:63-75.

30. DiLeo MV, Strahan GD, den Bakker M, Hoekenga OA. Weighted correlation network analysis (WGCNA) applied to the tomato fruit metabolome. PLoS One. 2011;6:e26683.

31. Basnet RK, Moreno-Pachon N, Lin K, Bucher J, Visser RGF, Maliepaard C, Bonnema G. Genome-wide analysis of coordinated transcript abundance during seed development in different Brassica rapa morphotypes. BMC Genomics. 2013;14:840.

32. Broderick SR, Wijeratne S, Wijeratn AJ, Chapin LJ, Meulia T, Jones ML. RNAsequencing reveals early, dynamic transcriptome changes in the corollas of pollinated petunias. BMC Plant Biol. 2014;14:307.

33. Smita S, Katiyar A, Pandey DM, Chinnusamy V, Archak S, Bansal KC. Identification of conserved drought stress responsive gene-network across tissues and developmental stages in rice. Bioinformation. 2013;9:72-8.

34. Borges RM, Bessière J-M, Ranganathan $Y$. Diel variation in fig volatiles across syconium development: making sense of scents. J Chem Ecol. 2013;39:630-42.

35. Tsamardinos I, Brown LE, Aliferis CF. The max-min hill-climbing Bayesian network structure learning algorithm. Mach Learn. 2006;65:31-78.

36. Moschen S, Bengoa Luoni S, Paniego NB, Hopp HE, Dosio GAA, Fernandez $P$, Heinz RA. Identification of candidate genes associated with leaf senescence in cultivated sunflower (Helianthus annuus L.). PLoS One. 2014;9:e104379.

37. Moschen S, Bengoa Luoni S, Di Rienzo J, Caro M, Tohge T, Watanabe M, Hollmann J, González S, Rivarola M, García-García F, Dopazo J, Hopp HE, Hoefgen R, Fernie A, Paniego N, Fernández P, Heinz R. Integrating transcriptomic and metabolomic analysis to understand natural leaf senescence in sunflower. Plant Biotechnol journal. 2015. DOI: 10.1111/pbi.12422.

38. Fernandez P, Soria M, Blesa D, DiRienzo J, Moschen S, Rivarola M, Clavijo BJ, Gonzalez S, Peluffo L, Príncipi D, Dosio G, Aguirrezabal L, García-García F, Conesa A, Hopp E, Dopazo J, Heinz RA, Paniego N. Development, characterization and experimental validation of a cultivated sunflower (Helianthus annuus L.) gene expression oligonucleotide microarray. PLoS One. 2012;7:1-11.

39. Smyth G. Limma: linear models for microarray data. In: Gentleman R, Carey V, Dudoit S, Irizarry R, Huber W, editors. Bioinforma Comput Biol Solut using R Bioconductor. New York: Springer; 2005. p. 397-420.

40. R Core Team. R: A language and environment for statistical computing. $R$ Foundation for Statistical Computing,. ISBN 3-900051-07-0. 2012.

41. Pérez-Rodríguez P, Riaño-Pachón DM, Corrêa LGG, Rensing SA, Kersten B, Mueller-Roeber B. PInTFDB: updated content and new features of the plant transcription factor database. Nucleic Acids Res. 2010;38(Database issue):D822-7.

42. Roessner-Tunali U, Hegemann B, Lytovchenko A, Carrari F, Bruedigam C, Granot D, Fernie AR. Metabolic profiling of transgenic tomato plants overexpressing hexokinase reveals that the influence of hexose phosphorylation diminishes during fruit development. Plant Physiol. 2003;133:84-99.

43. Peluffo L, Lia V, Troglia C, Maringolo C, Norma P, Escande A, Esteban Hopp H, Lytovchenko A, Fernie AR, Heinz R, Carrari F. Metabolic profiles of sunflower genotypes with contrasting response to Sclerotinia sclerotiorum infection. Phytochemistry. 2010;71:70-80.

44. Luedemann A, Strassburg K, Erban A, Kopka J. TagFinder for the quantitative analysis of gas chromatography-mass spectrometry (GC-MS)-based metabolite profiling experiments. Bioinformatics. 2008;24:732-7.

45. Langfelder P, Horvath S. WGCNA: an R package for weighted correlation network analysis. BMC Bioinformatics. 2008;9:559.

46. Shannon P, Markiel A, Ozier O, Baliga NS, Wang JT, Ramage D, Amin N, Schwikowski B, Ideker T. Cytoscape: a software environment for integrated models of biomolecular interaction networks. Genome Res. 2003;13:2498-504.

47. Tsamardinos I, Rakhshani A, Lagani V. Performance-Estimation Properties of Cross-Validation-Based Protocols with Simultaneous Hyper-Parameter Optimization. In: Artif Intell Methods Appl. 8445th edition. Lecture Notes in Computer Science. 2014. p. 1-14.

48. Breeze E, Harrisona E, McHattiea S, Hughesa L, Hickmana R, Hilla C, Kiddle S, Kim Y, Penfold CA, Jenkins D, Zhang C, Morris K, Jenner C, Jackson S, Thomas B, Tabrett A, Legaie R, Moore JD, Wild DL, Ott S, Rand D, Beynon J, Denby K, Mead A, Buchanan-wollaston V. High-resolution temporal profiling of transcripts during Arabidopsis leaf senescence reveals a distinct chronology of processes and regulation. Plant Cell. 2011;23(March):873-94.
49. Gregersen PL, Holm PB. Transcriptome analysis of senescence in the flag leaf of wheat (Triticum aestivum L.). Plant Biotechnol J. 2007;5:192-206.

50. Li Z, Peng J, Wen X, Guo H. Gene Network A nalysis and Functional S tudies of Senescence-associated Genes R eveal Novel Regulators of Arabidopsis Leaf Senescence. J Integr Plant Biol. 2012;54:526-39.

51. Liu L, Zhou Y, Zhou G, Ye R, Zhao L, Li X, Lin Y. Identification of early senescence-associated genes in rice flag leaves. Plant Mol Biol. 2008;67:37-55.

52. Malhotra S, Sowdhamini R. Interactions Among Plant Transcription Factors Regulating Expression of Stress-responsive Genes. Bioinform Biol Insights. 2014;8:193-8.

53. Wang Z, Gerstein M, Snyder M. RNA-Seq: a revolutionary tool for transcriptomics. Nat Rev Genet. 2009;10:57-63.

54. Balibrea Lara ME, Gonzalez Garcia M-C, Fatima T, Ehness R, Lee TK, Proels R, Tanner W, Roitsch T. Extracellular invertase is an essential component of cytokinin-mediated delay of senescence. Plant Cell. 2004;16:1276-87.

55. Roitsch T, González M-C. Function and regulation of plant invertases: sweet sensations. Trends Plant Sci. 2004;9:606-13.

56. Smeekens S, Ma J, Hanson J, Rolland F. Sugar signals and molecular networks controlling plant growth. Curr Opin Plant Biol. 2010;13:274-9.

57. Wingler A, Von Schaewen A, Leegood RC, Lea PL, Quick PW. Regulation of leaf senescence by cytokinin, sugars, and light. Plant Physiol. 1998;116:329-35.

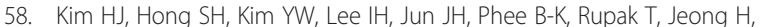
Lee $Y$, Hong BS, Nam HG, Woo HR, Lim PO. Gene regulatory cascade of senescence-associated NAC transcription factors activated by ETHYLENEINSENSITIVE2-mediated leaf senescence signalling in Arabidopsis. J Exp Bot. 2014:65:4023-36.

59. Kim JH, Nguyen NH, Jeong CY, Nguyen NT, Hong S-W, Lee H. Loss of the R2R3 MYB, AtMyb73, causes hyper-induction of the SOS1 and SOS3 genes in response to high salinity in Arabidopsis. J Plant Physiol. 2013;170:1461-5.

\section{Submit your next manuscript to BioMed Central and we will help you at every step:}

- We accept pre-submission inquiries

- Our selector tool helps you to find the most relevant journal

- We provide round the clock customer support

- Convenient online submission

- Thorough peer review

- Inclusion in PubMed and all major indexing services

- Maximum visibility for your research

Submit your manuscript at www.biomedcentral.com/submit
Biomed Central 